\title{
Protective effects of dexmedetomidine on intestinal ischemia-reperfusion injury
}

\author{
YONG SUN $^{1}$, QIANG GAO ${ }^{1}$, NAN WU $^{2}$, SHENG-DE LI $^{2}$, JING-XIN YAO ${ }^{2}$ and WEN-JIE FAN ${ }^{2}$ \\ Departments of ${ }^{1}$ Surgery and ${ }^{2}$ Anesthesia, Qingdao Women and Children's Hospital, \\ Qingdao, Shandong 266034, P.R. China
}

Received July 10, 2014; Accepted February 9, 2015

DOI: $10.3892 /$ etm.2015.2561

\begin{abstract}
Dexmedetomidine (DEX) has been hypothesized to possess anti-oxidative properties that may mitigate the damage caused by ischemia-reperfusion (IR) injury. The aim of the present study was to examine the effects of DEX on intestinal contractile activity, inflammation and apoptosis following intestinal IR injury. Intestinal IR injury was induced in rats by complete occlusion of the superior mesenteric artery for $60 \mathrm{~min}$, followed by a $60-\mathrm{min}$ reperfusion period. Rats received an intraperitoneal injection of $25 \mu \mathrm{g} / \mathrm{kg} \mathrm{DEX}$ at $30 \mathrm{~min}$ prior to the mesenteric IR injury. Following reperfusion, segments of the terminal ileum were rapidly extracted and transferred into an isolated organ bath. The contractile responses to receptor-mediated acetylcholine (Ach) and non-receptor-mediated potassium chloride $(\mathrm{KCl})$ were subsequently examined. Nitric oxide (NO) levels were determined and the expression levels of tumor necrosis factor (TNF)- $\alpha$, interleukin (IL)-6, Bax and Bcl-2 were measured using an enzyme-linked immunosorbent assay. The levels of telomerase and caspase- 3 were determined using reverse transcription-quantitative polymerase chain reaction. The results indicated that DEX treatment produced a significant reduction in the IR-induced contractile response to Ach and $\mathrm{KCl}$ in the intestinal tissue. Furthermore, DEX appeared to significantly ameliorate intestinal IR injury, in addition to reducing the production of NO. Similar reductions were observed in the intestinal expression levels of TNF- $\alpha$ and IL-6. In addition, DEX treatment resulted in a reduction in the expression levels of Bax in the intestinal tissues, while increasing those of $\mathrm{Bcl}-2$, in addition to significantly increasing the mRNA levels of telomerase and caspase-3. Therefore, the present study indicated that NO, TNF- $\alpha$ and IL- 6 may partially contribute to the pathogenesis of intestinal IR injury
\end{abstract}

Correspondence to: Dr Wen-Jie Fan, Department of Anesthesia, Qingdao Women and Children's Hospital, 217 Liaoyang West Road, Qingdao, Shandong 266034, P.R. China

E-mail: fanwenjie2016@yeah.net

Key words: dexmedetomidine, ischemia-reperfusion, inflammation, apoptosis in addition to the increased expression levels of Bax, Bcl-2, telomerase and caspase-3. These findings suggest that DEX possesses beneficial anti-apoptotic and anti-inflammatory effects in intestinal tissue following bowel injury.

\section{Introduction}

Intestinal ischemia-reperfusion (IR) is a common symptom of various diseases, including acute mesenteric ischemia, small bowel transplantation, abdominal aortic aneurysm, hemorrhagic, traumatic or septic shock and severe burn wounds (1). Numerous mediators and processes are involved in the pathogenesis of IR-induced intestinal injury, including reactive oxygen/nitrogen species, pro-inflammatory cytokines and leukocyte adhesion/infiltration (2-3). It is established that apoptosis and inflammation are increased significantly during IR in the gut and may serve key functions in the pathogenesis of IR-induced intestinal injury. Intestinal IR models have been used frequently in the study of apoptosis (4). There is a requirement for the identification of experimental agents that may be administered as adjunctive therapy to surgery in order to mitigate intestinal IR injury (5-7). However, these experimental agents may not be administrable during surgery or in the intensive care unit (ICU) (8). Therefore, the effects of anesthetic and sedative agents on IR injury may be significant factors in patient outcomes and thus require further study.

Dexmedetomidine (DEX) is a potent and selective $\alpha 2$ adrenergic receptor agonist. Clinically, DEX has been used as an adjunct to anesthesia, analgesia and ICU sedation (9). In addition, DEX offers good perioperative hemodynamic stability and reduces intraoperative anesthetic requirements; a number of prior studies have demonstrated that DEX reduces intestinal IR injury $(10,11)$. However, the effects of DEX on IR-associated reduced contractility of intestinal smooth muscle remains unclear, and there are a limited number of studies addressing the effects of DEX on apoptosis and inflammation in IR-induced intestinal injury.

The aim of the present study was to investigate the effects of DEX on intestinal contractile activity, inflammation and apoptosis in a rat model of IR-induced intestinal injury. These effects were examined via the evaluation of acetylcholine (Ach) and potassium chloride $(\mathrm{KCl})$-induced contractile responses. In addition, the protein levels of nitric oxide (NO), 
tumor necrosis factor (TNF)- $\alpha$, interleukin (IL)-6, Bax and Bcl-2, and the mRNA expression levels of telomerase and caspase-3 were determined.

\section{Materials and methods}

TNF- $\alpha$ and IL-6 enzyme-linked immunosorbent assay (ELISA). ELISA kits were purchased from Nanjing Jiancheng Bioengineering Institute (Nanjing, China) and all other reagents were purchased from commercial sources.

Animals. Male Sprague-Dawley rats (age range, 2-2.5 months) were purchased from the animal facility of Qingdao Medical University, Qingdao, China. The present study was approved by the local Medical Ethics Committee of Qingdao Women and Children's Hospital, Qingdao, China. Rats were reared under standard laboratory conditions $\left(22 \pm 2^{\circ} \mathrm{C}, 60 \pm 10 \%\right.$ relative humidity and a 12-h light-dark cycle) and had free access to food and water, but fasted overnight prior to the experiments.

Induction of IR injury. Rats were anesthetized intraperitoneally (i.p.) with ketamine (100 mg/kg; Sigma-Aldrich, St. Louis, MO, USA) and chlorpromazine (0.75 mg/kg; Sigma-Aldrich). Following induction of anesthesia, the abdomen was opened with a midline abdominal incision. Intestinal IR injury was produced by complete occlusion of the superior mesenteric artery followed by a period of reperfusion. The superior mesenteric artery was clamped for $60 \mathrm{~min}$. Following $60 \mathrm{~min}$ of ischemia, the vascular clamp at the superior mesenteric artery was removed and three drops of 2\% lidocaine (Sigma-Aldrich) were applied directly to the superior mesenteric artery to facilitate reperfusion. Blood circulation was restarted for a 60-min reperfusion period. Following the reperfusion period, the rats were euthanized with an overdose of ketamine $(200 \mathrm{mg} / \mathrm{kg})$ and chlorpromazine $(1 \mathrm{mg} / \mathrm{kg})$. A $20-\mathrm{cm}$ incision was made $1 \mathrm{~cm}$ distal to the ileocecal junction. The internal cavity was exposed and feces were cleaned, then washed with phosphate-buffered saline (PBS; Sigma-Aldrich) and dried. A mucosal smear of the small intestine was collected with a glass slide.

Experimental protocol. Rats were selected at random and divided into three groups ( $\mathrm{n}=10$ in each group) as follows: Sham group rats underwent an abdominal incision and their organs were exposed for $120 \mathrm{~min}$, but without clamping of the mesenteric artery, in order to distinguish the differences between the effects of intestinal IR and those of non-specific surgical stress; IR group rats received an i.p. injection of normal saline $(10 \mathrm{ml} / \mathrm{kg}) 30 \mathrm{~min}$ prior to the intestinal IR; and IR + DEX group rats received an i.p. injection of $25 \mu \mathrm{g} / \mathrm{kg}$ DEX dissolved in normal saline 30 min prior to the intestinal IR. The dose of DEX administered was based on a previous study (10).

The animals in the IR and IR + DEX groups, to which normal saline or DEX were applied, were euthanized $2.5 \mathrm{~h}$ after the injections. The sham group rats were euthanized $2 \mathrm{~h}$ after the sham operation in order to imitate the conditions of the rats in the IR group. Subsequent to the experiments, samples from the jejunum were collected from the animals for examination. Immediately following euthanasia, the rat intes- tinal tissues were rapidly removed and cleaned with PBS and frozen in liquid nitrogen (Tiangen Biotech Co. Ltd., Beijing, China). Tissues were homogenized $(10 \% \mathrm{wt} / \mathrm{vol})$ in ice-cold $10 \mathrm{mM}$ phosphate buffer ( $\mathrm{pH}$ 7.4), sonicated (Ultrasonic Instruments, Misonix, MI, USA) on ice for $15 \mathrm{sec}$ and centrifuged at $3,000 \mathrm{xg}$ for $20 \mathrm{~min}$. The resulting supernatants were collected for biochemical assays.

Preparation of terminal ileum. Ileal longitudinal muscle contractile activity was evaluated in isolated ileal segments following 1-h reperfusion in an organ bath (12). Strips of longitudinal muscle were removed $1 \mathrm{~cm}$ cephalad of the ileocecal junction. Strips were longitudinally suspended under a 2-g load in an organ bath containing $20 \mathrm{ml} \mathrm{Kreb's} \mathrm{solution} \mathrm{(in}$ $\mathrm{mM}: \mathrm{NaCl}, 118.5 ; \mathrm{KCl}, 4.8 ; \mathrm{KH}_{2} \mathrm{PO}_{4}, 1.2 ; \mathrm{MgSO}_{4} .7 \mathrm{H}_{2} \mathrm{O}, 1.2$; $\mathrm{CaCl}_{2}, 1.9 ; \mathrm{NaHCO}_{3}, 25$; and glucose, 10.1). The solution was continually gassed with a mixture of $5 \% \mathrm{CO}_{2}$ and $95 \% \mathrm{O}_{2}$ and maintained at $3^{\circ} \mathrm{C}$. After 60 -min equilibration with a 2-g load, Ach was added to the organ bath fluid at a final concentration of $10^{-6} \mathrm{M}$. Following 60 -min equilibration with a 2 -g load, $\mathrm{KCl}$ was added to the organ bath separately at a final concentration of $30 \mathrm{mM}$, in order to measure changes in the contractile responses of the samples. In the preparation of high $\mathrm{K}^{+}$solutions, $\mathrm{NaCl}$ was exchanged for equimolar amounts of $\mathrm{KCl}$, in order to maintain the physiological osmolarity of the Kreb's solution. Drugs were prepared daily in distilled water and stored in ice during the course of the experiments. Isometric force was monitored with an external force displacement transducer using a BL-420F Data Acquisition \& Analysis System (Chengdu Taimeng Science and Technology Co., Ltd., Chengdu, China).

Measurement of $\mathrm{NO}$ levels. The tissue levels of nitrite $\left(\mathrm{NO}_{2}{ }^{-}\right)$ and nitrate $\left(\mathrm{NO}_{3}{ }^{-}\right)$were measured in order to estimate $\mathrm{NO}$ production, as $\mathrm{NO}_{2}{ }^{-}$and $\mathrm{NO}_{3}{ }^{-}$are stable $\mathrm{NO}$ oxidative metabolites. Quantification of $\mathrm{NO}_{2}{ }^{-}$and $\mathrm{NO}_{3}^{-}$was based on the Griess reaction, in which a chromophore with a strong absorbance at $550 \mathrm{~nm}$ is formed by the reaction of $\mathrm{NO}_{2}^{-}$with a mixture of $0.01 \%$ naphthyl ethylenediamine, $1 \%$ sulfanilamide and $5 \%$ $\mathrm{H}_{3} \mathrm{PO}_{4}(5,7,8)$. The results are expressed as $\mu \mathrm{mol} / \mathrm{g}$ protein.

ELISA analysis of IL-6, TNF- $\alpha, B c l-2$ and Bax levels. IL-6, TNF- $\alpha, B c 1-2$ and Bax expression levels in the intestinal tissues were detected using commercial ELISA kits (Tiangen Biotech). The association between optical density (OD) and cytokine concentration was defined using the standard curve according to the manufacturer's instructions. These expression levels are expressed as $\mathrm{pg} / \mathrm{ml}$.

Reverse transcription-quantitative polymerase chain reaction (RT-qPCR) analysis of telomerase and caspase-3. Total RNA from the jejunum was isolated using TRIzol reagent (Gibco Life Technologies, Carlsbad, CA, USA) according to the manufacturer's instructions (13). RNA was quantified by measuring the OD at $260 \mathrm{~nm}$ and reverse transcribed into single-stranded cDNA using a RevertAid H Minus First Strand cDNA Synthesis kit (\#K1632; Thermo Fisher Scientific, Pittsburgh, PA, USA). The cDNA was amplified for 35 cycles in order to maintain the PCR product in the linear range. The PCRs were performed at $94^{\circ} \mathrm{C}$ for $5 \mathrm{~min}$, followed by 
Table I. Primer sequences for mRNA fragments amplified by quantitative polymerase chain reaction.

\begin{tabular}{ll}
\hline Gene & \multicolumn{1}{c}{ Primer sequences } \\
\hline Human telomerase reverse transcriptase & 5'-GCAGAATTCATGCCAGGGACTCCCCGCAGGTTG-3' \\
& 5'-CGGGTCGACTTACTCGTAGTTGAGGACGCTGAAC-3' \\
Caspase-3 & 5'-TGTCGATGCAGCTAACC-3' \\
& 5'-GGCCTCCACTGGTATCTTCTG-3' \\
$\beta$-Actin & 5'-GGAAATCGTGCGTGAC-3' \\
& 5'-GGAAGGTGGACAGTGAG-3' \\
\hline
\end{tabular}

A

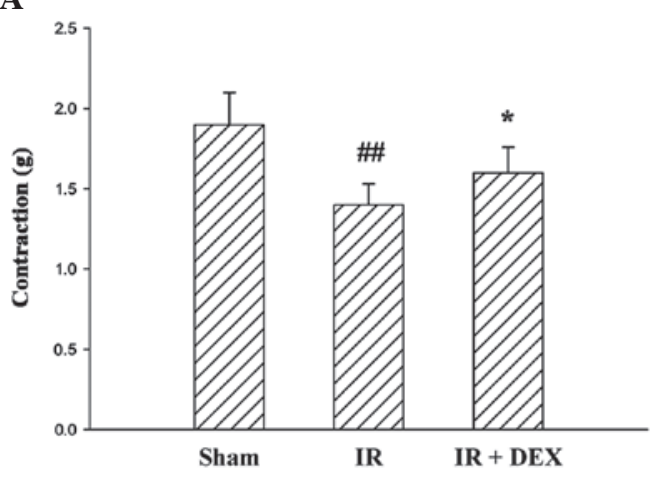

B

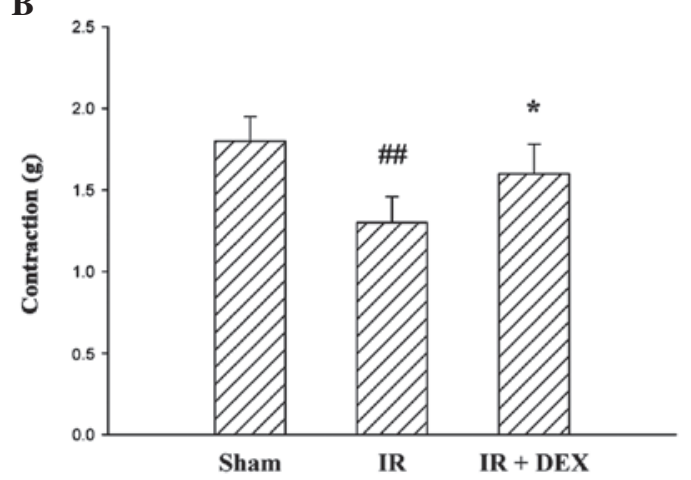

Figure 1. Effect of DEX on contraction response of smooth muscle to (A) $10^{-6} \mathrm{M}$ Ach and (B) $30 \mathrm{mM} \mathrm{KCl}$ in an intestinal IR model. Data are expressed as the mean \pm standard error $(\mathrm{n}=10) .{ }^{\#} \mathrm{P}<0.01$ vs. the sham group and ${ }^{*} \mathrm{P}<0.05$ vs. the IR group. No significant difference was observed between the sham and $\mathrm{DEX}$ groups. DEX, dexmedetomidine; Ach, acetylcholine; $\mathrm{KCl}$, potassium chloride; IR, ischemia-reperfusion.

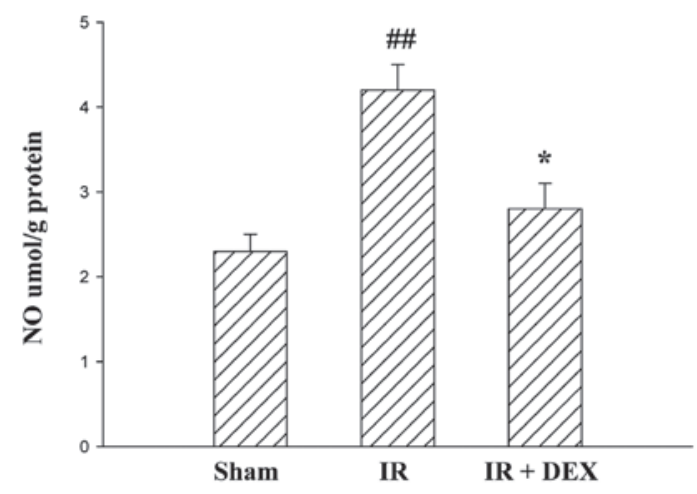

Figure 2. NO production in the three groups. A significant increase in NO levels was observed in the IR group compared with the sham group. By contrast, DEX preconditioning induced a marked reduction in NO levels compared with the IR group. Data are expressed as the mean \pm standard error $(n=10)$. ${ }^{\# \#} \mathrm{P}<0.01$ vs. the sham group and ${ }^{*} \mathrm{P}<0.05$ vs. the IR group. No significant difference was observed between the sham and DEX groups. NO, nitric oxide; DEX, dexmedetomidine; IR, ischemia-reperfusion.

35 cycles at $94^{\circ} \mathrm{C}$ for $30 \mathrm{sec}$, annealing at the corresponding temperature (Table I) for $30 \mathrm{sec}$ and $72^{\circ} \mathrm{C}$ for $30 \mathrm{sec}$, and a final extension at $72^{\circ} \mathrm{C}$ for $8 \mathrm{~min}$. PCR was performed using a Golden Easy PCR System (\#KT221; Tiangen Biotech) with gene-specific primers for $\beta$-actin, telomerase and caspase- 3 (Table I). $\beta$-Actin was used as an internal control to confirm mRNA integrity. The identities of all PCR products were confirmed by size, based on the known length of the DNA sequence on $1 \%$ agarose gel (Tiangen Biotech) stained by ethidium bromide (Tiangen Biotech). The OD was analyzed using a GeneSnap system (version 2.0; Syngene, Frederick, MD, USA).

Statistical analysis. Values are expressed as the mean \pm standard error. One-way analysis of variance was used, followed by Fisher's least-significant difference test for the homogeneity testing of variance (Levene's test). Data were analyzed using Dunnett's T3 test for the heteroscedasticity of variance test. $\mathrm{P}<0.05$ was considered to indicate a statistically significant difference. All statistical analyses were conducted using SPSS software, version 13.0 for Windows (SPSS, Inc., Chicago, IL, USA).

\section{Results}

DEX inhibits IR-induced attenuation of ileal longitudinal muscle contractility. The contractile responses induced by Ach were significantly inhibited by the induction of IR $(\mathrm{P}<0.01$; Fig. 1A). Administration of DEX at a dose of $25 \mu \mathrm{g} / \mathrm{kg}$ resulted in a significant reduction in the inhibition of contractility observed due to IR $(\mathrm{P}<0.05)$.

The addition of $\mathrm{KCl}$ at a final concentration of $30 \mathrm{mM}$ into the organ bath fluid resulted in the contraction of the terminal ileum segment (Fig. 1B). The contraction obtained from the tissues of rats in the IR group was reduced compared with the sham group rats $(\mathrm{P}<0.01)$. The contractile response of the $\mathrm{IR}+$ DEX group rats following treatment with $30 \mathrm{mM} \mathrm{KCl}$ was similar to that of the rats in the sham group. The administration of DEX $(25 \mu \mathrm{g} / \mathrm{kg})$ to the IR + DEX group resulted in 
A

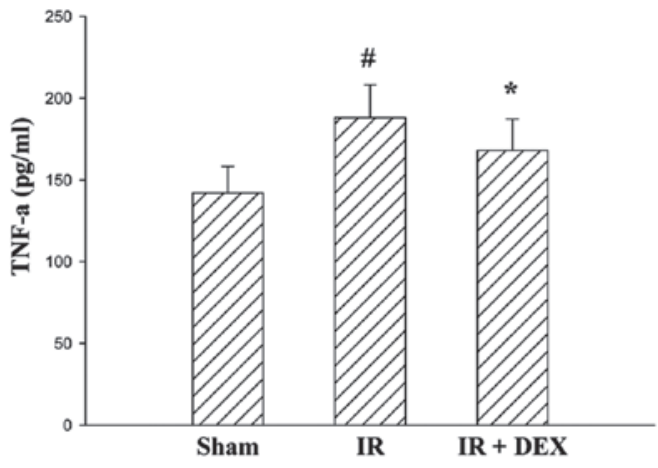

C

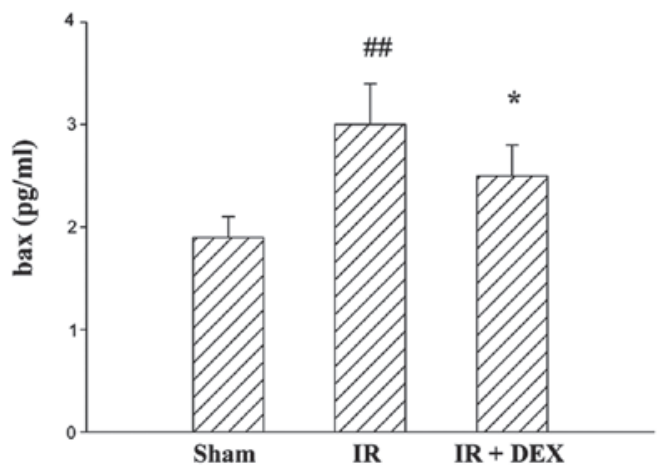

B

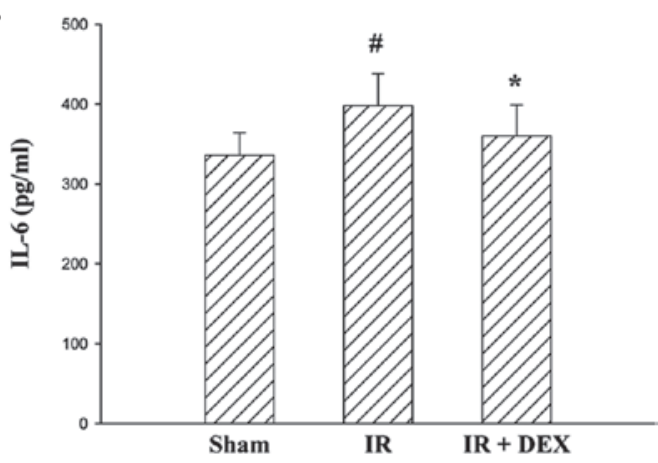

D

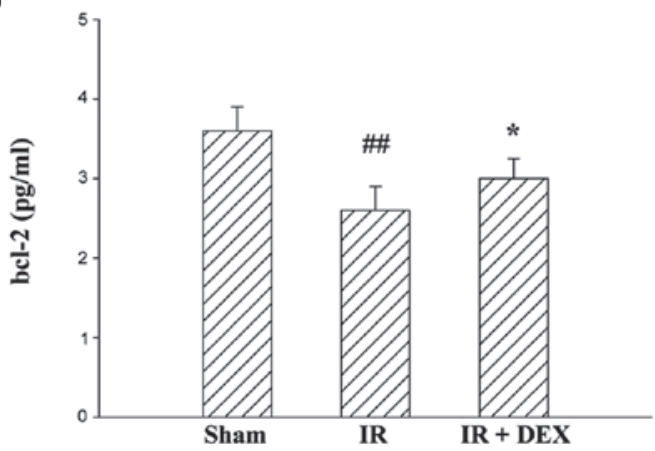

Figure 3. Effects of DEX administration on (A) TNF- $\alpha$, (B) IL-6, (C) Bax and (D) Bcl-2 content in the small intestine. Data are expressed as the mean \pm standard error $(\mathrm{n}=10)$. ${ }^{\#} \mathrm{P}<0.05,{ }^{\# \#} \mathrm{P}<0.01$ vs. the sham group and ${ }^{*} \mathrm{P}<0.05$ vs. the IR group. No significant difference was observed between the sham and $\mathrm{DEX}$ groups. DEX, dexmedetomidine; TNF- $\alpha$, tumor necrosis factor- $\alpha$; IL-6, interleukin-6; IR, ischemia-reperfusion.
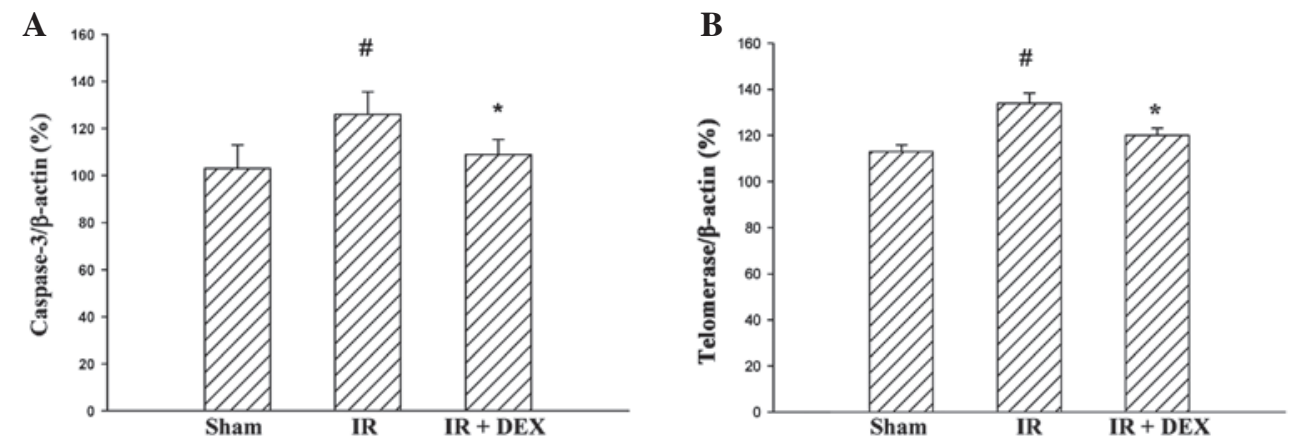

Figure 4. Effects of DEX on IR-induced mRNA expression of (A) caspase-3 and (B) telomerase in the small intestine measured by quantitative polymerase chain reaction. Data are expressed as the mean \pm standard error $(n=10) .{ }^{\#} \mathrm{P}<0.05$ vs. the sham group and ${ }^{*} \mathrm{P}<0.05$ vs. the IR group. No significant difference was observed between the sham and DEX groups. DEX, dexmedetomidine; IR, ischemia-reperfusion.

an amelioration of the $\mathrm{KCl}$-induced contractile response in the ischemic tissue $(\mathrm{P}<0.05)$.

$D E X$ reduces $I R$-induced increase in intestinal NO levels. The concentrations of NO in intestinal tissues of the groups are presented in Fig. 2. A significant increase in NO levels was observed in the IR group compared with the sham group $(\mathrm{P}<0.01)$. By contrast, the IR $+\mathrm{DEX}$ group exhibited a notable reduction in $\mathrm{NO}$ levels compared with the IR group $(\mathrm{P}<0.05)$.

Levels of TNF- $\alpha, I L-6$ and protein expressions of Bax and Bcl-2 on intestinal IR injury. To further assess intestinal IR injury, levels of TNF- $\alpha$, IL-6, Bax and Bcl-2 were also analyzed (Fig. 3). Compared with the sham group, the expression levels of TNF- $\alpha$, IL-6 $(\mathrm{P}<0.05)$ and $\mathrm{Bax}(\mathrm{P}<0.01)$ were significantly increased in the IR group rats; and this increase was significantly inhibited in the IR + DEX group rats $(\mathrm{P}<0.05)$. Furthermore, a reduction in tissue $\mathrm{Bcl}-2$ level was observed following intestinal IR injury. The Bcl-2 content was significantly higher in the IR + DEX treated group compared with the IR group $(\mathrm{P}<0.05)$. These results indicate that the DEX pretreatment markedly attenuated intestinal IR injury.

DEX suppresses the IR injury-induced increase in levels of telomerase and caspase-3 in the jejunum. The effects of DEX on intestinal cell survival following IR injury were investigated by analyzing the transcriptional levels of associated genes (telomerase and caspase-3) following DEX pretreatment. The mRNA expression levels of telomerase and caspase-3 were enhanced in the IR group compared with the sham group $(\mathrm{P}<0.05$; Fig. 4). Pretreatment with DEX resulted in a significant reduction in the mRNA expression levels 
of telomerase and caspase-3 compared with the IR group $(\mathrm{P}<0.05)$.

\section{Discussion}

The present study evaluated the effects of DEX pretreatment in small intestinal tissue with IR-induced injury. The results demonstrated that intestinal IR damage led to a reduction in ileal contractility in response to Ach (receptor-mediated) and $\mathrm{KCl}$ (non-receptor-mediated) induction. Administration of $25 \mu \mathrm{g} / \mathrm{kg}$ DEX appeared to mitigate this reduction in contractile response. Intestinal IR induces an inflammatory response within the area of muscle cells that results in the recruitment and extravasation of leukocytes into the smooth muscle syncytium (14). Reactive oxygen species and inflammatory leukocytes are reportedly involved in the progression of intestinal IR-induced remote organ injury (15). NO, as a toxic metabolite, may serve a key function in the initiation of intestinal mucosal injury (15). NO is beneficial as a messenger or modulator; however, under conditions such as oxidative stress, NO is potentially toxic. High levels of exogenous NO exert a cytopathic effect on the intestine that increases the extent of mucosal injury (15-17). Potoka et al (15) suggested that peroxynitrite may be able to induce enterocyte apoptosis via a number of mechanisms, including the inhibition of mitochondrial function, adenosine triphosphate depletion, activation of caspases via cytochrome $c$, mitochondrial release of apoptosis-activating factor-1 and the activation of poly(ADP-ribose) synthetase. Additionally, peroxynitrate may influence the inhibition of enterocyte proliferation and differentiation in the intestinal crypts by interfering with tyrosine kinase signaling cascades. NO, through its toxic metabolite peroxynitrite, serves a major role in the initiation of intestinal mucosal injury in clinical conditions associated with sustained inducible NO synthase upregulation in the gut $(16,17)$. In the present study, NO levels of the IR group rats were significantly higher compared with the sham group rats. In addition, the NO levels of the IR + DEX group were significantly reduced compared with the IR group. Thus, the results indicated that NO is a critical mediator of the inflammatory response during the development of intestinal injury, and that DEX significantly reduces intestinal tissue levels of NO.

Previous studies have demonstrated that DEX inhibits the expression of a number of inflammatory mediators, including NO, prostaglandin E2, TNF- $\alpha$ and IL-6 (18). In previous studies, treatment with DEX led to a reduction in the expression levels of TNF- $\alpha$ in ischemic hippocampal tissue, and a reduction of the TNF- $\alpha$ and IL- 6 concentrations in endotoxin-exposed rats (19,20). Furthermore, a prior study demonstrated a similar reduction in TNF- $\alpha$ and IL-6 levels in an experimental spinal cord injury (21). These experimental data were supported by clinical studies demonstrating reduced TNF- $\alpha$ and IL- 6 levels in critically ill patients with sepsis or postoperative major surgery $(22,23)$. Therefore, the anti-inflammatory effects of DEX may be responsible for the prevention of the mesenteric artery occlusion induced by IR injury. The results of the current study indicated that intestinal IR injury triggers the production of pro-inflammatory cytokines and that DEX pretreatment prevents this production. Intestinal IR injury has been demonstrated to induce a significant increase in IL-6 levels in intestine samples, which is consistent with the results of the present study. Furthermore, the results of the present study are consistent with prior studies (19), suggesting that DEX exhibits anti-inflammatory effects.

In addition, the present study suggests that DEX exerts a protective and anti-apoptotic effect against intestinal ischemic injury. To the best of our knowledge, there are no previous studies regarding the effects of DEX on apoptosis in IR-induced intestinal injury. Proteins of the Bcl-2 family, as anti-apoptotic proteins, induce and integrate cell survival and death signals, associated with apoptosis in cells. By contrast, the increased expression of the pro-apoptotic protein Bax mediates an enhanced rate of apoptosis. In the present study, the expression levels of Bax and the labeling index of caspase-3, a key caspase in the apoptotic pathway, were markedly reduced in the intestinal tissues of the IR + DEX group rats. Upregulation of Bcl-2 and DEX, however, downregulated the expression of Bax.

The expression levels of telomerase reverse transcriptase (TERT) significantly increase following stimulation of the cerebellar fastigial nucleus (FN). TERT may bind to Bax and inhibit Bax-mediated apoptosis by suppressing the mitochondrial relocalization of Bax from the cytosol (24). Telomerase is an enzyme that adds a six-base DNA repeat sequence (TTA GGG) to chromosome ends and thereby prevents their shortening during successive rounds of mitosis (25). In the present study, IR induced telomerase activity in rat intestine samples. RT-qPCR analyses demonstrated that the mRNA expression levels of telomerase and caspase-3 were significantly reduced in the DEX group compared with the IR group. These results indicate that DEX treatment may inhibit IR-induced damage by modulating the expression of telomerase and caspase-3.

In conclusion, the protective effects of DEX in the intestine may be due to its anti-inflammatory and anti-apoptotic properties. The results of the present study indicated that the downregulation of telomerase and caspase- 3 mRNA may be involved in the protective effect of DEX against IR-induced damage. Further studies are required to clarify the possible mechanisms underlying this protective effect.

\section{References}

1. Shen J, Fu G, Jiang L, Xu J, Li L and Fu G: Effect of dexmedetomidine pretreatment on lung injury following intestinal ischemia-reperfusion. Exp Ther Med 6: 1359-1364, 2013.

2. Xia G, Martin AE, Michalsky MP and Besner GE: Heparin-binding EGF-like growth factor preserves crypt cell proliferation and decreases bacterial translocation after intestinal ischemia/reperfusion injury. J Pediatr Surg 37: 1081-1087, 2002

3. El Assal ON and Besner GE: Heparin-binding epidermal growth factor-like growth factor and intestinal ischemia-reperfusion injury. Semin Pediatr Surg 13: 2-10, 2004.

4. Fujise T, Iwakiri R, Wu B, Amemori S, Kakimoto T, Yokoyama F, Sakata Y, Tsunada S and Fujimoto K: Apoptotic pathway in the rat small intestinal mucosa is different between fasting and ischemia-reperfusion. Am J Physiol Gastrointest Liver Physiol 291: G110-G116, 2006.

5. Taha MO, Miranda-Ferreira R, Fagundes AL, Fagundes DJ, Simões RS, Santos JM, Souza PD, Oliveira-Júnior IS, Marchini A, Gomes IT, et al: Effects of L-nitro-arginine methyl ester, an inhibitor of nitric oxide biosynthesis, on intestinal ischemia/reperfusion injury in rabbits. Transplant Proc 42: 457-460, 2010.

6. Yang S, Chou WP and Pei L: Effects of propofol on renal ischemia/reperfusion injury in rats. Exp Ther Med 6: 1177-1183, 2013. 
7. Petrat $\mathrm{F}$ and de Groot $\mathrm{H}$ : Protection against severe intestinal ischemia/reperfusion injury in rats by intravenous resveratrol J Surg Res 167: e145-e155, 2011.

8. Hanci V, Erol B, Bektaş S, Mungan G, Yurtlu S, Tokgöz H, Can M and Ozkoçak Turan I: Effect of dexmedetomidine on testicular torsion/detorsion damage in rats. Urol Int 84: 105-111, 2010.

9. Lai YC, Tsai PS and Huang CJ: Effects of dexmedetomidine on regulating endotoxin-induced up-regulation of inflammatory molecules in murine macrophages. J Surg Res 154: 212-219, 2009

10. Lin S, Dai N, Cheng Z, Shao W and Fu Z: Effect of dexmedetomidine-etomidate-fentanyl combined anesthesia on somatosensory- and motor-evoked potentials in patients undergoing spinal surgery. Exp Ther Med 7: 1383-1387, 2014.

11. Kiliç K, Hanci V, Selek S, Sözmen M, Kiliç N, Citil M, Yurtlu DA and Yurtlu BS: The effects of dexmedetomidine on mesenteric arterial occlusion-associated gut ischemia and reperfusion-induced gut and kidney injury in rabbits. J Surg Res 178: 223-232, 2012

12. Ozacmak VH, Sayan H, Arslan SO, Altaner S and Aktas RG: Protective effect of melatonin on contractile activity and oxidative injury induced by ischemia and reperfusion of rat ileum. Life Sci 76: 1575-1588, 2005.

13. Gao Y, Deng XG, Sun QN and Zhong ZQ: Ganoderma spore lipid inhibits N-methyl-N-nitrosourea-induced retinal photoreceptor apoptosis in vivo. Exp Eye Res 90: 397-404, 2010.

14. Hassoun HT, Weisbrodt NW, Mercer DW, Kozar RA, Moody FG and Moore FA: Inducible nitric oxide synthase mediates gut ischemia/reperfusion-induced ileus only after severe insults J Surg Res 97: 150-154, 2001.

15. Potoka DA, Nadler EP, Upperman JS and Ford HR: Role of nitric oxide and peroxynitrite in gut barrier failure. World J Surg 26 806-811, 2002

16. Upperman JS, Potoka D, Grishin A, Hackam D, Zamora R and Ford HR: Mechanisms of nitric oxide-mediated intestinal barrier failure in necrotizing enterocolitis. Semin Pediatr Surg 14: $159-166,2005$
17. Szadujkis-Szadurska K, Grzesk G, Szadujkis-Szadurski L, Gajdus M and Matusiak G: Role of nitric oxide and cGMP in the modulation of vascular contraction induced by angiotensin II and Bay K8644 during ischemia/reperfusion. Exp Ther Med 5: 616-620, 2013

18. Lin CY, Tsai PS, Hung YC and Huang CJ: L-type calcium channels are involved in mediating the anti-inflammatory effects of magnesium sulphate. Br J Anaesth 104: 44-51, 2010.

19. Eser O, Fidan H, Sahin O, Cosar M, Yaman M, Mollaoglu H, Songur A and Buyukbas S: The influence of dexmedetomidine on ischemic rat hippocampus. Brain Res 1218: 250-256, 2008.

20. Taniguchi T, Kurita A, Kobayashi K, Yamamoto K and Inaba H: Dose- and time-related effects of dexmedetomidine on mortality and inflammatory responses to endotoxin-induced shock in rats. J Anesth 22: 221-228, 2008.

21. Can M, Gul S, Bektas S, Hanci V and Acikgoz S: Effects of dexmedetomidine or methylprednisolone on inflammatory responses in spinal cord injury. Acta Anaesthesiol Scand 53: 1068-1072, 2009.

22. Memiş D, Hekimoğlu S, Vatan I, Yandim T, Yüksel M and Süt N: Effects of midazolam and dexmedetomidine on inflammatory responses and gastric intramucosal $\mathrm{pH}$ to sepsis, in critically ill patients. Br J Anaesth 98: 550-552, 2007.

23. Venn RM, Bryant A, Hall GM and Grounds RM: Effects of dexmedetomidine on adrenocortical function, and cardiovascular, endocrine and inflammatory responses in post-operative patients needing sedation in the intensive care unit. Br J Anaesth 86: 650-656, 2001

24. Yang Y, Liu JL, Qin C, Li JP, Wang XL, Zhang ZX and Zhang L: Effects of cerebellar fastigial nucleus electrical stimulation on telomerase reverse transcriptase expression and mitochondrial apoptotic pathway in rats with focal cerebral ischemia and reperfusion.Zhonghua Yi Xue Za Zhi 91: 1643-1648, 2011 (In Chinese).

25. Lau BW, Wong AO, Tsao GS, So KF and Yip HK: Molecular cloning and characterization of the zebra fish (Danio rerio) telomerase catalytic subunit (telomerase reverse transcriptase, TERT). J Mol Neurosci 34: 63-75, 2008. 